\title{
Sistema de administración de servicios para empresas de taxi
}

\author{
Service management system for taxi companies
}

${ }^{1}$ Elizabeth del Carmen López Vásquez

${ }^{2}$ Wilson César Callisaya Choquecota

\section{RESUMEN}

En la actualidad, la industria de taxi ha crecido de manera descontrolada, es por ello que, para ayudar a una gestión eficaz de dicho servicio, se ha venido implementando diversas aplicaciones de software. En el presente trabajo se describió una aplicación móvil desarrollada para que las empresas puedan administrar sus servicios de taxi, detallando las diferentes funcionalidades implementadas y el impacto actual de dicho software; así como la experiencia obtenida en el desarrollo de un sistema de administración de servicios para taxis y su impacto en el mercado. Del mismo modo, se mencionó los inconvenientes encontrados en la etapa de desarrollo y finalmente se describió su funcionamiento.

Palabras clave: Android, Aplicaciones móviles, App, e-hail, Taxiapp.

\section{ABSTRACT}

At present, the Taxi industry has grown in an uncontrolled manner, which is why, in order to help the efficient management of this service, various software applications have been implemented. This paper describes a mobile application, developed so that companies can manage their taxi services, detailing the different functionalities implemented and the current impact of said software; as well as the experience gained in the development of a service administration system for taxis and the impact on the market. At the same time, the inconveniences found in the development stage are mentioned and finally its operation is described.

Keywords: Android, Movil aplication, App, e-hail, Taxiapp,.

'Escuela de Posgrado. Universidad Nacional Jorge Basadre Grohmann. Tacna-Perú. E-mail: elizalopezvasquez@gmail.com ${ }^{2}$ Escuela de Posgrado. Universidad Alas Peruanas. Tacna-Perú. E-mail: 


\section{INTRODUCCIÓN}

En el Perú existe una gran diversidad de empresas de taxi y radio taxi, las cuales, debido a la gran competencia, desean ser elegidas en el mercado de su rubro y ganarse la fidelización de los clientes para tener mayor posicionamiento; lo que las motiva a dar cada vez más un mayor valor agregado a sus empresas.

Asimismo, hoy en día, vemos el uso de Smartphones en todo lugar y la mayoría de personas ya empiezan a depender de ellos, así como muchos dependían de las páginas web (Cumbe y Neira, 2012).

Siendo la seguridad un tema relevante a nivel mundial, una aplicación que pueda darnos información del taxista es una herramienta muy útil para el pasajero (Arias y Loor, 2012).

En la actualidad, diferentes empresas a nivel mundial han desarrollado estas aplicaciones para solicitar un servicio de taxi, buscando conectar a los pasajeros y taxistas. Por ejemplo, en este rubro encontramos a empresas como: Hailo, Uber, Lift, Tappsi Easy Taxi, Safer Taxi, Way Taxi, GrabTaxi, CabiFy, Ommm, Smart taxi, 99taxi, TaxiBeat, TaxiYa, entre otros, tal como se aprecia en la Figura 1, y podemos notar que el mercado está en alza y empieza una carrera por cubrir dicho mercado de las TaxiApp (Hailo) (Designing for labour).
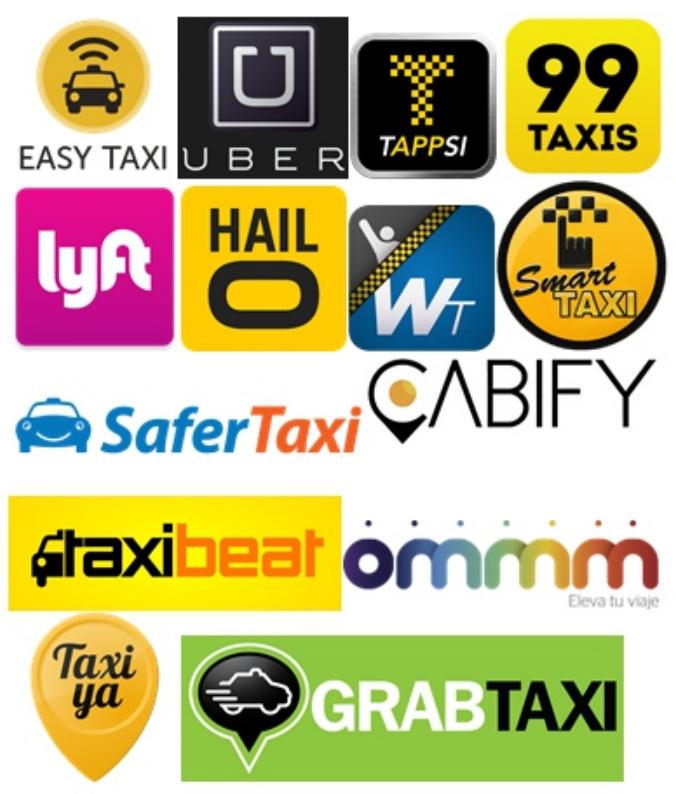

Figura 1. Ejemplos de empresas que ofrecen una aplicación de servicio de Taxi.
Un análisis en el mercado de las aplicaciones de taxis en Hong Kong muestra que este tipo de negocio es prometedor y tiene un gran potencial para ser desarrollado en aplicaciones. Sin embargo, el mercado de taxis se encuentra todavía en transición, aún se puede observar una gran resistencia al cambio.

Con la creciente toma de conciencia y beneficios únicos de las aplicaciones de taxi, es muy probable que se pueda reemplazar los servicios de radiotaxi en los próximos años (Chan, Chang, Lau, Law \& Lei, 2016).

El crecimiento de este mercado de aplicaciones para taxis ha sido verificado por la Comisión de taxis y limosinas de la ciudad de Nueva York (The New York City Taxi and Limousine Commission, 2015)

Además, solamente es necesario ver el crecimiento de Tappsi durante el 2012 en Bogotá, como indica la Figura 2 (Diario La República, 2013).

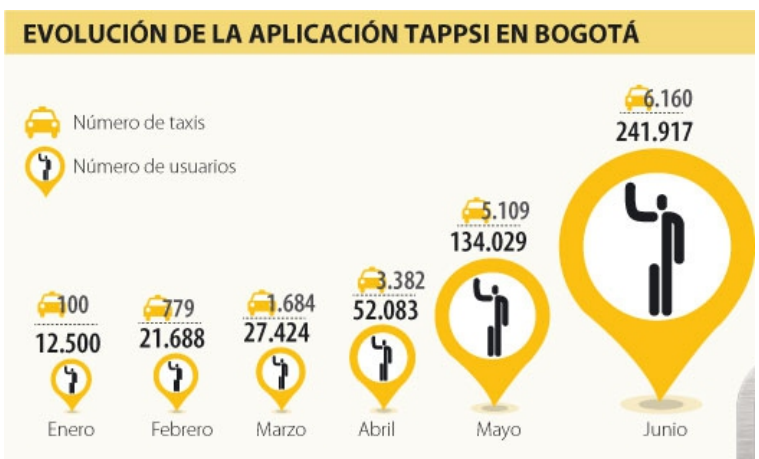

Figura 2. Evolución de la aplicación Tappsi en Bogotá. Fuente: Diario La República (2013)

Optimizar la asignación de las solicitudes de servicio de taxis maximizaría la utilidad de los vehículos, reduciendo el periodo de inactividad durante su jornada. Además, se puede aprovechar la gestión para minimizar el tiempo de espera de sus clientes y los costos de operación, lo cual proporcionaría una ventaja competitiva con respecto a los taxis particulares que buscan sus pasajeros en el camino o los de taxi con radioperadora, pues el taxi vería las peticiones en cola según su posición geográfica, estas son unas cuantas bondades que nos puede ofrecer una aplicación de este tipo (Murillo).

Pero no bastaría una sólida aplicación sino un eficiente plan de mercadeo para que dicho emprendimiento siga en el mercado. Una investigación de Yong (2015) sugiere que para 
este negocio funcione, se debe enfatizar y promover las ventajas y beneficios de las aplicaciones móviles de taxis para tener una percepción positiva sobre ellos. Además, el investigador sugiere que se debe educar a los conductores para asegurarse de que conocen las ventajas y beneficios, a su vez los taxistas deben comprometerse al uso de este tipo de aplicación.

Este tipo de aplicación solo sería el principio, debido a que se pueden brindar más servicios, por ejemplo, el de compartir el taxi o un servicio de colectivo. Para ello, se necesitará más algoritmos que puedan indicar una ruta óptima para el viaje. Además, gracias a los datos obtenidos, se podría hasta sugerir una ruta para que los taxistas puedan encontrar a sus pasajeros según los datos de otros taxistas (gracias a la minería de datos), intentando dar un enfoque diferente al mercado $u$ otorgando más beneficios al conductor, pero siempre partiendo de este primer enfoque: un servicio de conexión de pasajero y taxista por medio del celular Smartphone (Vaishali, Sonali, Priyanka, 2015) (Thakkar, Bhosale, Gawade \& Mehta, 2015)

Todo ello ha motivado el desarrollo del presente trabajo, que muestra el sistema de administración de servicios para empresas de taxi. Dicho sistema de administración se personaliza para cada empresa que lo solicite, tanto las funcionalidades consideradas como los problemas que aparecieron en su desarrollo, así como nuestro mercado actual.

\section{MATERIALYMÉTODOS}

\section{Presentación Problema y Objetivo}

Existe una necesidad en las empresas de taxi para poder administrar sus unidades, algunos problemas frecuentes son el desconocimiento de las posiciones de todas las unidades, falta de más canales de comunicación diferentes a la radio base, presencia nula en dispositivos móviles, seguridad reducida para el pasajero al ignorar el taxi que lo atenderá, desconocimiento de vías para llegar a un destino por parte de taxistas nuevos, estos son algunos de los problemas que presenta una empresa de taxi.

Todo ello ha motivado el desarrollo de un aplicativo que pueda apoyar tanto a los pasajeros y taxistas, así como a la gerencia para poder administrar sus servicios de taxi.

\section{Antecedentes del Estudio}

Hay diversos trabajos que nos hablan sobre la arquitectura, construcción y desarrollo de este tipo de proyectos en años anteriores.

Cumbe y Neira (2012) indican que la aplicación debería:

- Tener un listado de todas las empresas de taxi de la ciudad, de tal modo que al hacer clic se llame directamente a la central para solicitar el servicio.

- Precisar de un mapa, donde se muestre a todos los taxistas, y al hacer clic, se podría solicitar el servicio de dicho taxi.

Por su parte, Arias y Loor (2012) indican que la aplicación debería:

- Permitir publicar en las redes sociales, sea Facebook o Twitter, las notificaciones de solicitud de servicio. Por otro lado, debe permitir enviar un correo electrónico al pasajero.

- Poder acceder a una base de datos interna y externa de datos del usuario.

- También debe tener acceso a un mapa virtual para conocer su posición y enviar esta posición al servidor.

- Escanear códigos QR para verificar que efectivamente el taxista que viene es el solicitado.

- Facilitar un botón de llamada de Auxilio, el cual envíe correos a las cuentas registradas y lo publique en redes sociales.

- Poder enviar notificaciones de SMS para cualquier eventualidad.

Rodriguez (s/f) indica que la aplicación debería:

- Registrar al pasajero y al taxista.

- Gestionar las peticiones de los pasajeros a los taxistas.

- Calificar el servicio de taxi.

- Eliminar cuentas.

- Modificar datos personales.

Castro, Franco e Ibarra (2014) indican que la aplicación debería:

- Estar disponible en la PlayStore y en la AppStore.

- Poder reportar casos de emergencias a las autoridades.

- Informar en un tiempo máximo de 10 
segundos la aceptación de un taxista.

- Poder solicitar un taxi remotamente.

- Solicitar un registro único para su uso, tanto taxistas y pasajeros.

- Poseer un menú para poder explorar sus funcionalidades.

- Almacenar el estado del pasajero, de tal manera que no tenga que iniciar sesión cada vez que desee usar la aplicación.

- No almacenar información personal en el dispositivo sino en la base de datos en la nube.

Por otro lado, Quintana (2011) indica que la aplicación debería poseer los siguientes módulos:

- Módulo de seguridad: En el cual se podrán actualizar los perfiles, registrar nuevos usuarios y asignar acceso a las funcionalidades por perfil.

- Módulo de configuración de servicio: Que permitirá detallar los tipos de unidades de servicio, marcas, generar zonas, dar de alta y de baja a una unidad de servicio.

- Módulo de Servicio de Transporte: Que permitirá dar de alta o de baja a un chofer, asignar a un chofer a una unidad de servicio, listar los servicios priorizándolos por fecha y hora, permitir escoger una petición, permitir asignar un pedido a un taxista para que lo atienda.

- Módulo de Reportes: Permitirá mostrar la cantidad de servicios por empresa, zona, unidad de servicio o por chofer, permitirá mostrar información sobre tarifas promedio del mercado, permitirá mostrar un reporte de pedidos aceptados y rechazados, permitirá mostrar un reporte de los imprevistos ocurridos, permitirá un reporte de las unidades asignadas por fecha, permitir ver los rankings.

- Módulo del cliente: Permitirá ingresar su pedido, especificando su dirección de origen y de destino, también la fecha y hora de recojo, permitir ver ofertas de los taxistas a su vez del tiempo aproximado, permitir elegir a un taxista, permitir la visualización de todos los pedidos realizados a su vez podrá ver la hora y el taxista que lo atendió, permitir ver los gastos incurridos en los servicios.

Seesan, Rungkasiri \& Cooharojananone (2012) indican que la aplicación debería:

- Realizar alertas de emergencia de una manera rápida y sencilla

- Reportar el comportamiento no deseado de los taxistas.
- Una aplicación que pueda calcular las tarifas de taxi.

- Ayudar a determinar la ruta ideal para llegar al destino.

- Compartir información de la experiencia de viajes con otros.

- Permitir realizar llamadas a la central.

Como se puede apreciar, hay varias investigaciones que hablan de los requerimientos de este tipo de sistema, cada uno con su propio enfoque. A continuación, se describen las funcionalidades consideradas para un proyecto de este tipo, así como problemas y mercado actual en la siguiente sección.

\section{Descripción de la Propuesta}

\section{- $\quad$ Funcionamiento}

Consiste en 3 aplicaciones principalmente. Se puede ver la interacción de ellas en la Figura 3.

\section{EL APLICATIVO}

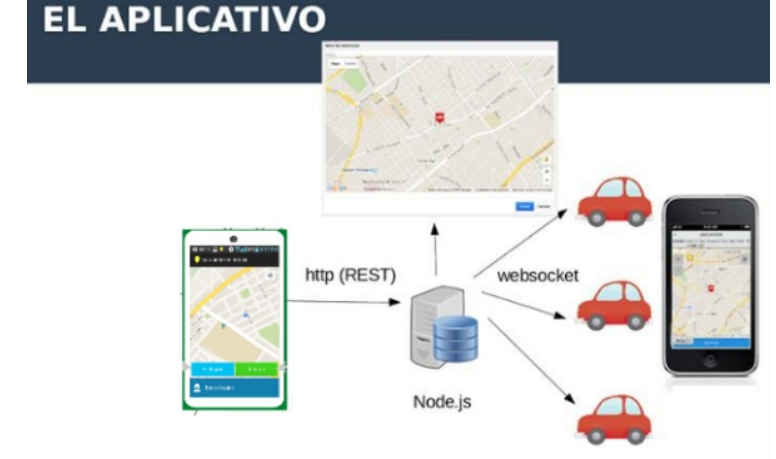

Figura 3. Funcionamiento de la propuesta

Una aplicación para el pasajero para realizar las peticiones, una aplicación para el taxista para la recepción de las peticiones, un servicio en la web para el registro, administración y control de los taxistas.

\section{Descripción del aplicativo}

\section{- Pasajero}

Una vez instalado el aplicativo, bastará ingresar el número de celular. Si no está registrado el número, le solicitará su nombre y apellido. Posterior a ello, la aplicación validará el número enviando un código de verificación por un SMS, informando también con un correo adicional a la administración. El sistema detectará su ubicación en un mapa virtual precisando la dirección, entonces, el pasajero podrá 
realizar la petición de un taxi, si desea puede añadir una nota de referencia. Una vez aceptada la petición por un taxista, se podrá ver el tiempo estimado que se demorará y la distancia, y en tiempo real podrá ver como se aproxima el taxista. Finalmente, podría recibir el sonido de una bocina virtual de parte del taxista cuando esté cerca.

\section{- Taxista}

Si el taxista pertenece a la empresa puede registrarse con sus datos en la administración. Se generará una contraseña de ingreso, la cual se le entregará al taxista si corresponde. También, la administración puede registrar a los taxistas por el panel web. Una vez activada la aplicación, el taxista podrá recibir notificaciones de solicitudes de pasajeros con un sonido característico de la aplicación, a pesar de no tener la aplicación en primer plano. Asimismo, el taxista podrá apreciar la distancia del pasajero que está dentro de la cobertura que él previamente definió. Una vez aceptada la solicitud, podrá visualizar a su objetivo en un mapa virtual. Si desea podrá hacer uso de herramientas de navegación como Google Maps o Waze, la cual entregará la ubicación del taxista y la de su objetivo. Al llegar a su destino, puede hacer uso de una bocina virtual para dar aviso de su llegada y finalmente presionar el abordar cuando el pasajero haya subido al vehículo. Adicionalmente, tiene opciones para indicar una situación de emergencia y poner marcadores en un mapa, informando a sus compañeros y a la administración sobre tráfico, operativos o eventos.

\section{- Administración}

La Administración dispone una sección de registro del taxista y de un listado de taxistas afiliados, del cual se puede expulsar a las unidades que no cumplan con las políticas de la empresa. A su vez, se puede ver en un mapa las peticiones realizadas por los pasajeros, tanto las exitosas como las fallidas y un mapa de monitoreo de taxistas, donde se podrá ver a los taxistas conectados, ocupados, con peticiones o desconectados. Desde el panel de administración, se podrá también aceptar peticiones, reenviar peticiones a todas las unidades, enviar mensajes a una o varias unidades, así como poner marcadores en el mapa virtual.

\section{Funcionalidades de la Aplicación}

\section{Pasajero}

Registro, verificando el número de celular por SMS.

Mapa virtual, donde se muestra la posición y dirección respectiva.

Botón para pedir taxi, y añadir más información como referencia.

Botón para llamar directamente a la oficina de la empresa.

Ver en tiempo real cómo el taxista se desplaza hasta su ubicación.

Ver la distancia aproximada donde está el taxi.

Ver el tiempo aproximado que demorará en llegar.

Historial de carreras realizadas.

Almacenar la última dirección en la misma calle, para que así el GPS pueda ubicarlo en un punto diferente o se actualice con la última dirección.

Recepción del sonido de bocina enviado por el taxista.

Datos completos del taxista que acepta la solicitud, incluyendo foto del taxista y del carro.

Baneo automático en abuso de cancelaciones.

La empresa, opcionalmente, podrá contemplar en su aplicativo de pasajero la opción de tarifario, indicando un destino. Este tarifario estará basado en la distancia.

\section{Taxista}

Historial de carreras realizadas

Datos de los pasajeros incluyendo la distancia de ellos.

Poder seleccionar la cobertura que uno desea atender.

Botón para indicar que está ocupado y no recibir más solicitudes.

Notificaciones con sonido cuando exista una nueva solicitud de un pasajero.

Tener la posibilidad de recibir estas notificaciones a pesar de estar en segundo plano.

Inicio de la aplicación cuando se enciende el celular.

Opción para añadir marcadores como operativo, tráfico, operativo municipal o eventos, enviando notificación a sus compañeros de ese nuevo marcador. Mapa virtual donde se muestra al pasajero que solicitó el servicio. 
Acceso a un navegador en un mapa donde se mostrará la ruta óptima para llegar y lo guiará a su destino.

Opción de enviar una bocina virtual, la cual llegará a su pasajero cuando llegue a su objetivo.

Distinción entre peticiones de la central o del aplicativo directo.

Recepción y envío de mensajes de texto por medio del aplicativo.

Botón de emergencia, el cual emitirá un sonido de notificación a sus compañeros y mostrará dicha ubicación en el mapa.

\section{Administrador}

Un panel para registro de taxistas.

Cuentas para diferentes tipos de acceso.

Opción de recuperar contraseña para los taxistas.

Listar a los taxistas registrados, con la posibilidad de expulsar a los taxistas que no cumplen las políticas de la empresa.

Mapa virtual de peticiones, donde se podrá ver las peticiones exitosas y fallidas de los pasajeros, mostradas por día, semana, mes.

Mapa virtual para monitorizar a los taxistas, en el cual se precisarán diferentes estados: conectado, ocupado, en servicio y desconectado.

Posibilidad de aceptar peticiones del aplicativo de pasajero desde el panel de administración, para no perder peticiones si faltara unidades en una zona.

Posibilidad de enviar peticiones a las unidades activas en el aplicativo.

Envío de mensajes personalizados a una o varias unidades.

Envío de petición a una sola unidad para poder realizar capacitaciones.

Envío de peticiones a grupos predeterminados.

Generación de marcadores operativos, tráfico o eventos.

Generación de recordatorios, que avisarán de una posible reserva a la central.

Reportes de peticiones por taxista y por empresa.

\section{RESULTADOS}

En el desarrollo de la presente aplicación se ha utilizado:

En Software: Android Studio para el desarrollo de las aplicaciones de celular tanto taxista y pasajero, usando el patrón modelo vista presentador, Sublime Text para la edición de código del server, Mongodb como de base de datos, node.js, bitbucket como repositorio de código, Trello para organización de tareas, Robomongo como SGBD, un hosting, un dominio, un proveedor de SMS, un proveedor servicio de correos electrónicos.

En Hardware: Computadoras I5 con memoria RAM superior a 4 gigabytes, diferentes equipos de celulares de diferentes empresas de telefonía y con sistema Android. Inicialmente, se empezó a operar para celulares con la versión de Android 2.3, ahora con soporte en la 4.0 a superior.

\section{Inconvenientes en la etapa de desarrollo}

- Problema de comunicación entre dispositivos, dado que inicialmente se usó la comunicación vía GCM (Google cloud Messaging) y luego, por un cambio en su documentación, se optó por usar sockets.

- Conseguir un proveedor de SMS que pueda dar soporte a todos los operadores.

- Lograr mantener el servicio de la aplicación del taxista siempre activo, dado que la mayoría de celulares eliminan servicios no prioritarios.

- Cambios en las nuevas opciones de los celulares para control de la batería, dado que eliminan servicios de forma automática si consideran que dichos servicios consumen mucha batería en pantalla inactiva.

- Lograr considerar un testeo eficiente en diferentes versiones y tamaños de celular.

- Cambios importantes en el IDE Android Studio, teniendo que reestructurar el proyecto.

- Disposición de los conocimientos de diferentes ámbitos de desarrollo (Api Rest, ciclo de vida de Android, manejo de sockets, express, node.js, Mongodb, uso de librerías, manejo de xml, plantillas ejs, manejo del api de google maps, etc.)

\section{Interacción de las diferentes interfaces del} aplicativo

\section{Pasajero}

Se dispone de una pantalla de login, la cual solicitará su número celular. Si es la primera vez, deberá registrarse con su nombre y apellido, se puede apreciar ello en la Figura 4. 


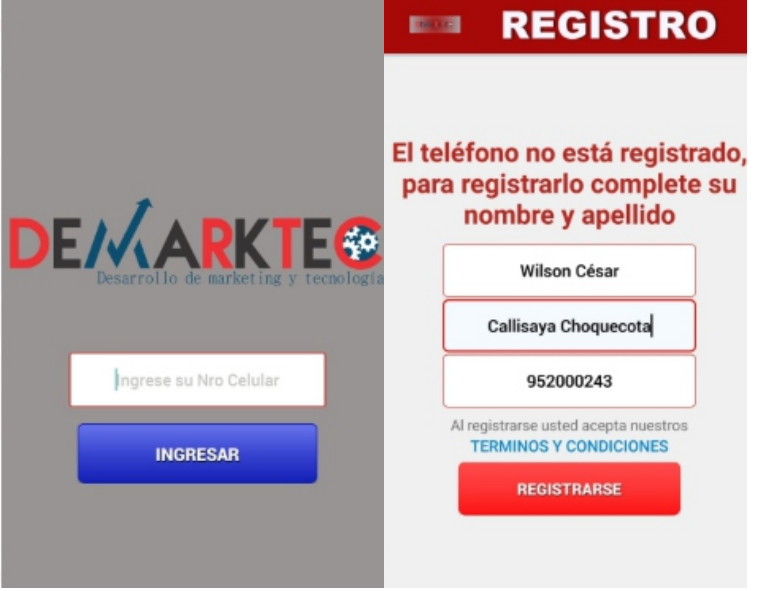

Figura 4. Interfaces para ingreso y registro.

Una vez registrado, le llegará un SMS de verificación a su número de celular. Eso también ocurrirá si ya estaba registrado, según se aprecia en la Figura 5.

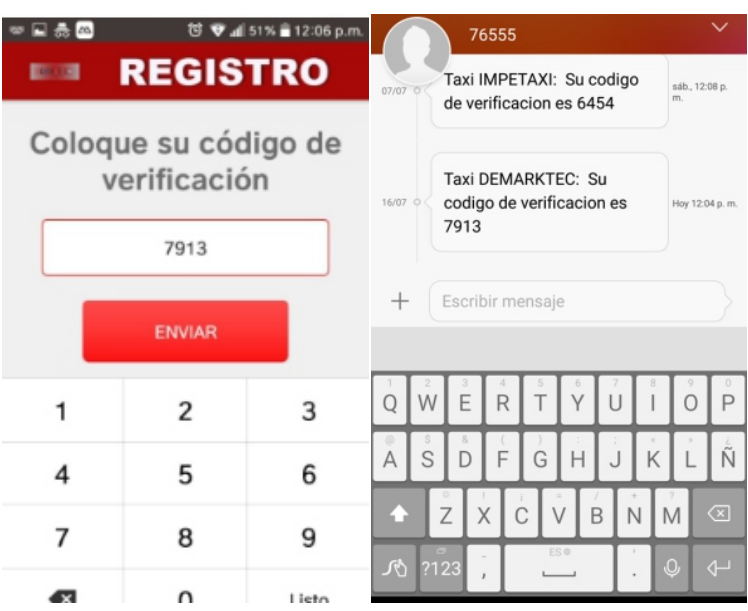

Figura 5. Interfaces de proceso de verificación de celular y recepción por SMS.

Una vez que se verificó el número de celular, podrá ver su ubicación en un mapa virtual, el cual indicará su dirección y podrá realizar una petición de taxi presionando el botón "Pedir Taxi”, si desea puede añadir una referencia adicional. En una ventana adicional podrá verificar si la información es correcta antes de realizar el pedido. Al presionar el botón de "Llamar taxi" se mostrará un cuadro de espera hasta que un taxi cercano acepte el pedido, este proceso se aprecia en la Figura 6.

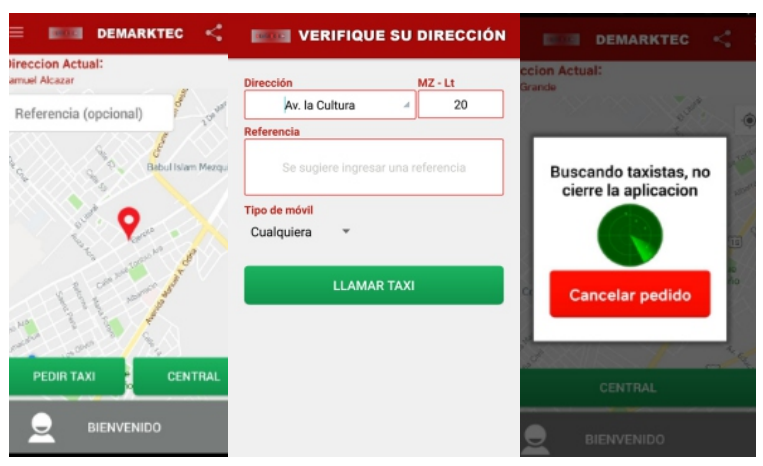

Figura 6. Interfaces de mapa virtual, de verificación de información y radar animado en espera a que un taxista acepte.

Una vez aceptada la solicitud por un taxista, se mostrará los datos de dichos taxistas: nombre, placa, teléfono, foto del chofer y su vehículo. Se podrá ver el tiempo estimado y la distancia en la cual se encuentra el taxi mientras espera, también podrá ver su desplazamiento en tiempo real. A su vez, en cualquier momento, el pasajero podrá ver todos los datos del taxista y llamarlo desde la aplicación. Este proceso se aprecia en la Figura 7.

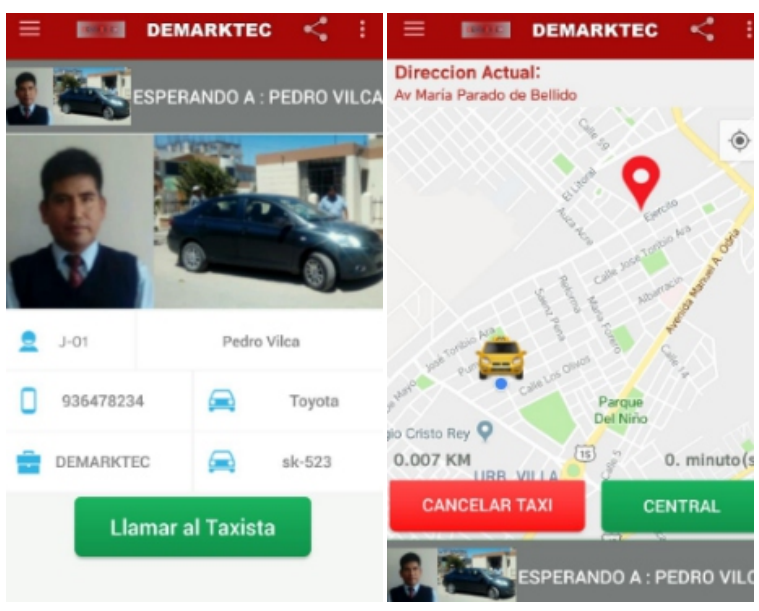

Figura 7. Interfaces de datos del taxista en aceptación del servicio, de espera y visualización detallada taxista que aceptó el servicio.

Si el taxista se encuentra cerca, podrá proceder a realizar el sonido de una bocina virtual y una vez a bordo del vehículo el pasajero podrá finalizar el servicio. Si el taxista finaliza el servicio, el pasajero podrá calificar el servicio realizado como se aprecia en la Figura 8. 


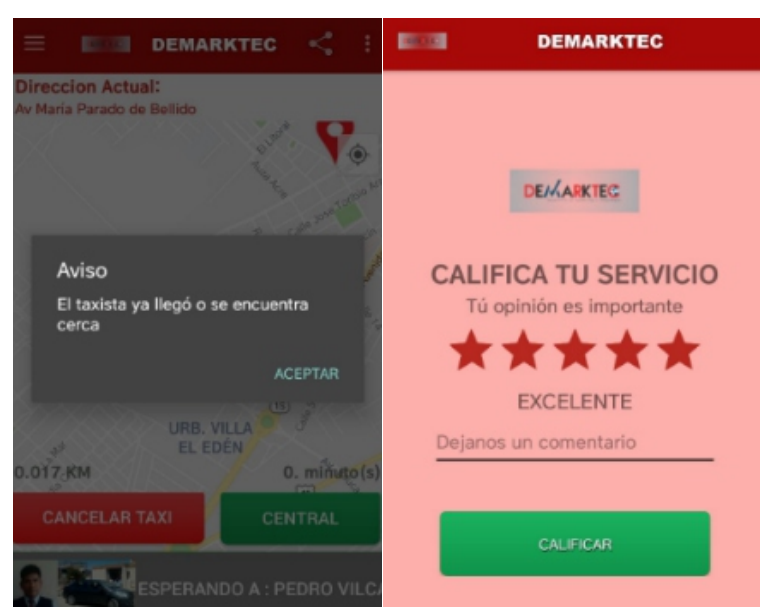

Figura 8. Interfaces de bocina virtual, de recorrido hacia el destino y de calificación del servicio.

\section{Taxista}

El taxista deberá registrarse y solicitar su contraseña a la administración para poder ingresar a la aplicación. Una vez haya ingresado al entorno de la aplicación, podrá observar la lista de peticiones en la cual se acumularán los pedidos solicitados de acuerdo a la cobertura que establezca de acuerdo a su preferencia. Dichas interfaces se pueden observar en la Figura 9.

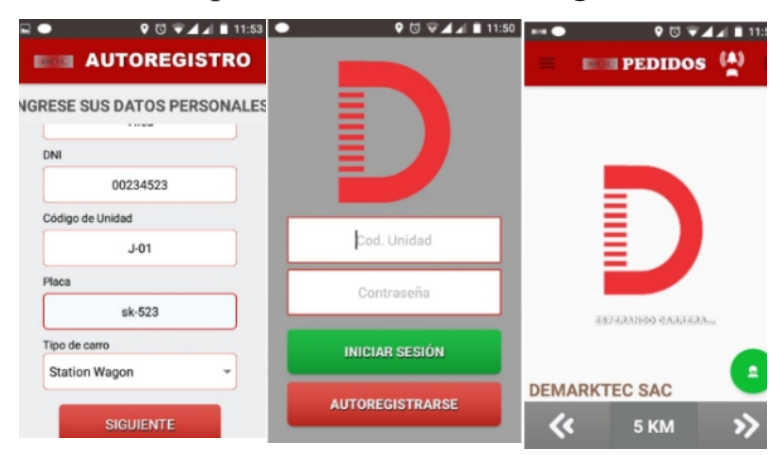

Figura 9. Interfaz de login del taxista, de registro y de la lista de peticiones.

Cuando un pasajero realiza la petición dentro de la cobertura seleccionada, el taxista lo verá en la lista de peticiones, donde se podrán observar los datos del pedido y la distancia del destino. Una vez aceptada la petición, se podrá ver al pasajero en un mapa con el cual podrá orientarse para llegar a su objetivo, los datos del pasajero estarán siempre disponibles. Lo descrito se aprecia en la Figura 10.

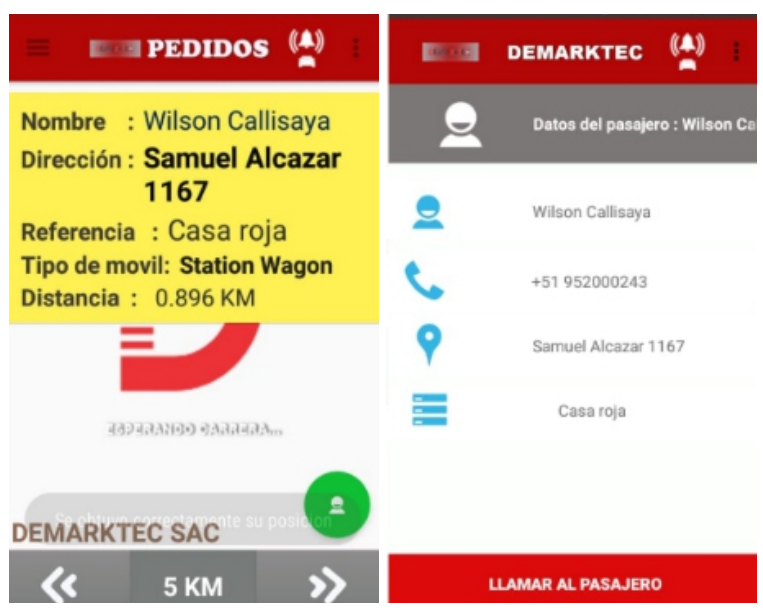

Figura 10. Interfaces de lista de peticiones con un pedido pendiente, de mapa virtual del taxista y de datos del pasajero cuando se está en un servicio.

Adicionalmente puede hacer uso de la navegación, la cual lo llevará a un entorno donde se le recomendará el mejor recorrido para llegar a su objetivo, el taxista también puede recibir y enviar mensajes a la central. Lo descrito se aprecia en la Figura 11.

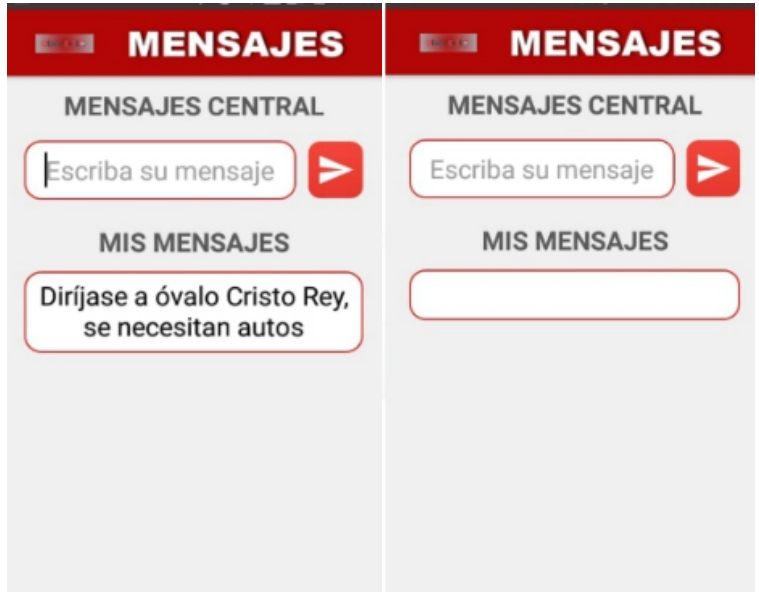

Figura 11. Interfaces de navegación y de mensajes a la central.

El taxista puede ver su historial de carreras y distinguir entre peticiones generadas por la central o desde una aplicación de pasajero. Adicionalmente, también puede generar marcadores como operativo, tráfico, eventos y operativo municipal. Lo descrito se aprecia en la Figura 12. 


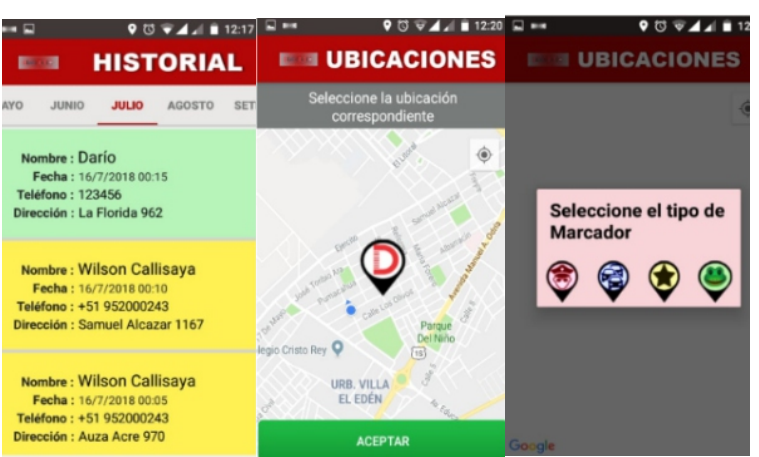

Figura 12. Interfaces de historial de peticiones y de marcadores.

\section{Administración}

La administración dispondrá de un entorno web, para su acceso deberá ingresar con su usuario y contraseña. Aquí se podrá ver a los taxistas registrados, como se aprecia en la Figura 13.

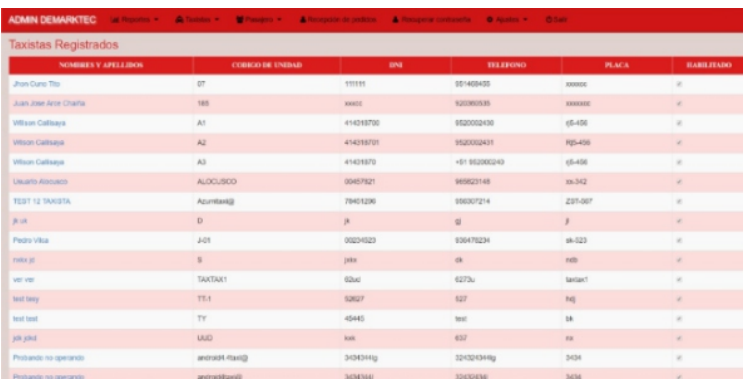

Figura 13. Interfaz del entorno web para la administración de taxis en la sección lista de taxistas registrados.

La administración podrá realizar el registro de taxistas y a su vez modificarlo; además de designar supervisores, los cuales también podrán ver las localizaciones de todas las unidades. A su vez, podrán expulsar a las unidades que no cumplan las políticas de la empresa. El panel de edición de datos de un taxista se aprecia en la Figura 14.

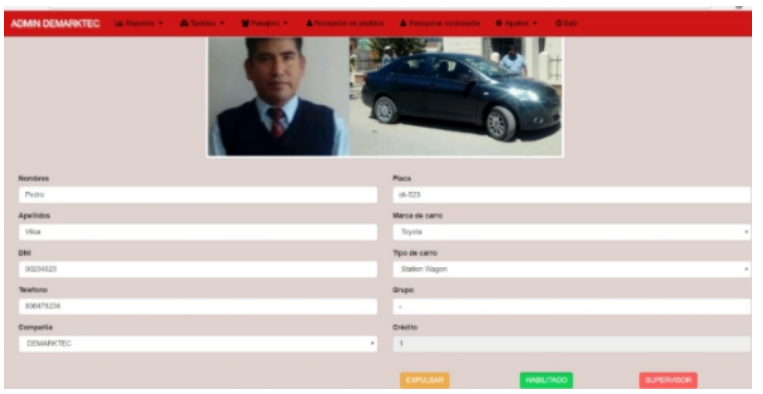

Figura 14. Interfaz del entorno web para la administración de taxis en la sección edición de taxistas.
La administración podrá interactuar con el aplicativo de pasajero y taxista, tanto para aceptar peticiones como para reenviar las solicitudes. Además, podrá guardar las peticiones generadas para que se llenen automáticamente al repetirlas, generar marcadores, enviar mensajes, generar recordatorios y monitorear todas sus unidades. El panel que permite realizar dichas operaciones se muestra en la Figura 15.

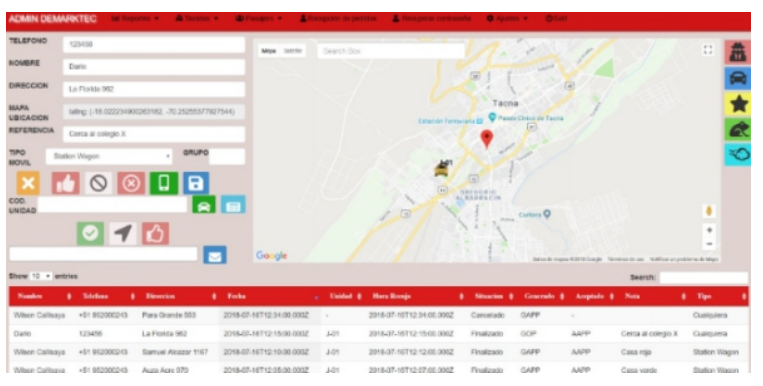

Figura 15. Interfaz del entorno web para la administración de taxis en la sección recepción de pedidos.

\section{DISCUSIÓN}

\section{Mercado actual del aplicativo}

Actualmente, el sistema de administración de servicios de taxi de nuestra propuesta está siendo usado por 27 empresas en 10 ciudades del Perú, como se muestra en la Tabla 1.

Tabla 1. Empresas de radio taxi que usan la propuesta del sistema de administración de taxi

\begin{tabular}{|c|c|c|}
\hline Ciudad & Empresas de Radio Taxi & $\begin{array}{l}\text { Total de } \\
\text { Empresas }\end{array}$ \\
\hline Tacna & Alo14 & 1 \\
\hline Moquegua & $\begin{array}{c}\text { Torvac } \\
\text { JAS, Panataxi, } \\
\text { Impetaxi, Taxisur, } \\
\text { Angels del Sur, Express } \\
\text { Reyna, Tatataxi, AQP, } \\
\text { Taxi Continental, Taxi }\end{array}$ & 1 \\
\hline Arequipa & $\begin{array}{c}\text { Lider } \\
\text { Turismo Cusco, Taxi }\end{array}$ & 10 \\
\hline Cusco & Seguro & 2 \\
\hline Madre de Dios & Turismo Madre de Dios & . \\
\hline Cajamarca & Plus, Taxis Unidos & 2 \\
\hline Chiclayo & $\begin{array}{l}\text { Radiotaxi Chiclayo } \\
\text { Mobil Express, Online }\end{array}$ & 1 \\
\hline Trujillo & $\begin{array}{c}\text { Corporation } \\
\text { Asetaxi, TaxiArchi, } \\
\text { Taxista Digital, Taxi Jet, } \\
\text { Taxi Vea, Taxi Vip }\end{array}$ & 2 \\
\hline Huancayo & Digital & 6 \\
\hline Ayacucho & Translorito & 1 \\
\hline \multicolumn{2}{|c|}{ Total de Empresas } & 27 \\
\hline
\end{tabular}




\section{CONCLUSIONES}

En este trabajo se ha presentado un sistema de administración de servicios de taxi; el cual, según diferentes investigadores, es un negocio rentable y es de gran interés para diferentes empresas de radiotaxi. Sin embargo, su desarrollo implica una cantidad de retos que debe afrontar el desarrollador, además que debe tenerlos en cuenta a la hora de implementar una aplicación similar, considerando las funcionalidades que sugieren diferentes autores.

Se recomienda ampliar funcionalidades para que el pasajero pueda escoger un taxista cercano, también se podría retirar la opción de cobertura para que esta sea dinámica y cambiante en el tiempo, desde el pasajero que solicita la petición. Dentro de este plan de actualización, se podría incluir la radio virtual, siendo una herramienta útil para las empresas de taxis. A su vez, otra posible actualización sería detectar y sincronizar las llamadas desde la plataforma web para que estas se escriban automáticamente si el cliente ya registrado solicita una unidad de taxi.

\section{AGRADECIMIENTO}

Los autores expresamos nuestro agradecimiento a 1a Escuela Profesional de Ingeniera en Informática y Sistema de la Universidad Nacional Jorge Basadre Grohmann-Tacna.

\section{REFERENCIAS}

CUMBE, Marisol y NEIRA, Washington. Aplicación para smarthphone de mapas y ubicaciones de buses y taxis de la ciudad de Guayaquil "Transpórtate en Guayaquil". Tesis de pregrado. Universidad Católica de Santiago de Guayaquil, Ecuador. 2012.

ARIAS, Oscar y LOOR, Josué. Modelo de negocios para el desarrrollo de una aplicación para dispositivos móviles para los usuarios del servicio de taxi de Guayaquil. Tesis de pregrado. Universidad Católica de Santiago de Guayaquil, Ecuador. 2012.

Hailo: The Digital Cab Hailing Disruption. $\mathrm{e} b$ o o k s . c a $\mathrm{p} \mathrm{g} \mathrm{e} \mathrm{m} \mathrm{i} \mathrm{n} \mathrm{i} \mathrm{-}$ consulting.com/dm/Hailo.pdf

Designing for labour: Uber and the On-Demand $\mathrm{Mob}$ i e Workfor ce. http://www.mobilelifecentre.org/sites/de fault/files/uber $\% 20$ final $\% 20$ camera $\% 20$ ready.pdf

CHAN, Jacky; CHANG, Vicky; LAU, William; LAW, Lawrence \& LEI, Corrine. Taxi App Market Analysis in Hong Kong. Journal of Economics, Business and Management, 4(3): 239-242, 2016. ISSN $2301-3567$. Disponible en: http://www.joebm.com/vol4/397A001.pdf

The New York City Taxi and Limousine $\mathrm{C}$ o m m i s s i o $\mathrm{n} \quad 2015$. http://www.nyc.gov/html/tlc/downloads/ pdf/ehail_q5_report_final.pdf.

La Republica. 2013. Disponible en: http://www.larepublica.co/consumo/enseis-meses-tappsi-realiz $\% \mathrm{C} 3 \% \mathrm{~B} 3$-dosmillones-de-carreras_43207

MURILlO, J. TaxiApp, San José, Costa Rica, D i s p o n i b l e e n http://bb9.ulacit.ac.cr/tesinas/publicacio nes/045756.pdf

YONG, William. Factors influencing Malaysian taxi drivers behavioral intention to adopt mobile taxi application. International Journal of Economics, Commerce and Management, 3 (11): 139-156, 2015. ISSN: 2348 0386. Recuperado de: http://ijecm.co.uk

VAISHALI, Ghumare; SONALI, Gandhakte \& PRIYANKA, Aher. Smart Traveller Proficient Taxi Business Application. International Journal for Scientific,3(7): 806-807, 2015. ISSN:2321-0613.

THAKKAR, Santosh; BHOSALE, Supriya, GAWADE, Namrata \& MEHTA, Sonia. Proposed Advance Taxi Recommender System Based On a Spatiotemporal Factor Analysis Model. International Journal of Application or Innovation in Engineering \& Managment,4(4):161166, 2015. ISSN: 2319-4847.

RODRIGUEZ, Andres. Aplicativo Móvil para la Administración de solicitudes de taxi, Pereira, Colombia, pp. 41. disponible en http://ribuc.ucp.edu.co:8080/jspui/bitstre $\mathrm{am} /$ handle/10785/1367/ANDR\%C3\%89 S\%20RODR\%C3\%8DGUEZ\%20ESCU DERO..pdf? sequence $=3$

CASTRO, Lizzeth; FRANCO, Luis y IBARRA, Santiago. Elaboración del estudio de prefactibilidad para el montaje de una empresa para ofrecer servicios de taxi seguro por medio de dispositivos móviles en la ciudad de Bogota, Trabajo de Grado. Escuela Colombiana de Ingeniería Julio Garavito - Unidad de Proyectos. Bogota, Colombia, 2014. 
QUINTANA, Hernán. Sistema de control, gestión y administración del servicio de Taxi. Tesis de pregrado. Pontificia Universidad Católica del Perú. 2011.

SEESAN, Ramana, RUNGKASIRI, Tippakorn \& COOHAROJANANONE Nagul. Taxi
Friend: Innovation Application for Taxi Passenger, en International Conference Innovative Research in a Changing and Challenging World. Phuket, Thailand. 2012. 